\title{
Indicadores clave de rendimiento en terminales de contenedores y su relación con la sostenibilidad ambiental. Aplicación al sistema portuario español
}

\author{
Key performance indicators in container terminals and their relationship with \\ environmental sustainability. Application to the spanish port system
}

\author{
María del Mar Gesé Bordils ${ }^{1} \quad$ Nicoletta González-Cancelas ${ }^{1} \quad$ Beatriz Molina Serrano ${ }^{1 *}$
}

Recibido 20 de junio de 2019, aceptado 23 de junio de 2021

Received: June 20,2019Ａccepted: June 23,2021

\begin{abstract}
RESUMEN
Los indicadores clave de desempeño de los procesos o KPI (Key Performance Indicator) son herramientas de gestión ampliamente utilizadas por las empresas en todo el mundo para medir y evaluar el desempeño de sus procesos y gestionarlos de la manera más eficaz y eficiente posible. En el ámbito portuario, la tendencia general en el análisis de la explotación de una terminal de contenedores es comparar los ratios y parámetros internacionales que aparecen en la bibliografía internacional sobre explotación y planificación portuaria, criterios con los que se explotan, y así establecer lo que una terminal se aleja o se acerca en su explotación a los valores de estos parámetros internacionales.

El objetivo de esta investigación es encontrar una relación entre los indicadores de productividad (KPI) y los indicadores de sostenibilidad en su ámbito medioambiental, de las terminales de contenedores del sistema portuario español. Se pretende encontrar una serie de indicadores para el estudio de las terminales de contenedores, desde el punto de vista de la gestión operativa y ambiental.

La principal conclusión de la investigación es que las terminales de contenedores del sistema portuario español, son heterogéneas tanto en la gestión de la productividad como en la ambiental por lo que es muy difícil establecer relaciones claras.
\end{abstract}

Palabras clave: Terminal de contenedores, KPI, sostenibilidad portuaria.

\begin{abstract}
Key Performance Indicators (KPI) are management tools widely used by companies around the world to measure and evaluate the performance of their processes and manage them in the most effective and efficient way possible. In the port areas, the general trend in the operation analysis of a container terminal is to compare the ratios and international parameters that appear in the international literature on port exploitation and planning. These criteria allow to work and to establish what operation of a terminal moves away or approaches from international parameters.

The objective of this research is to find a relationship between KPI and sustainability indicators in their environmental field, of container terminals, applied this to the Spanish Port System. The aim is to find a series of indicators from the point of view of operational and environmental management.

The main conclusion of the research is that the terminals of Spanish Port System are heterogeneous in productivity management and in environmental management, so it is very difficult to establish clear relationships.
\end{abstract}

Keywords: Container terminals, KPI, port sustainability.

1 Universidad Politécnica de Madrid. Departamento de Ingeniería Civil. Transportes. Madrid, España.

E-mail: magebo.93@gmail.com, nicoleta.gcancelas@upm.es, beatriz.molinas@alumnos.upm.es

* Autor de correspondencia: beatriz.molinas@alumnos.upm.es 


\section{ESTADO DEL CONOCIMIENTO}

\section{Introducción}

La palabra sostenibilidad admite múltiples definiciones, y casi siempre queda ligada a los conceptos de desarrollo sostenible y de responsabilidad social de las empresas [1]. Una de las definiciones más acertadas es: "Capacidad para mantener la continuidad en el largo plazo del medio ambiente y de las actividades humanas en sus aspectos económicos, sociales, institucionales y ambientales" [1].

Se entiende que en el marco de la gestión sostenible la aportación de valor no debe limitarse únicamente a la aportación de valor económico. El concepto de sostenibilidad aplicado a entidades sociales como municipios o países tiene un sentido claro [2], pues en principio parece lógico que el objetivo de los poderes públicos sea el mantenimiento y la potenciación a largo plazo del capital económico, ambiental y socio-cultural de los grupos humanos, es decir, la buena gestión de los recursos y su reparto social. En cambio, el enfoque para entidades particulares, como son las empresas o los individuos, es diferente: Si su único objetivo es el corto plazo, entonces la sostenibilidad no interesa en su gestión económica y aparece como un freno a sus intereses; sin embargo, si sus objetivos son generar valor económico a medio y largo plazo, aparecen otros valores en la gestión empresarial, donde el concepto de sostenibilidad es vital y, además, le permitirá controlar los costes no productivos, los riesgos, el compromiso social, su imagen de marca, así como innovar y abrirse a nuevos mercados.

El concepto de gestión sostenible, con la integración de las dimensiones sociales y ambientales en el gobierno y administración de sociedades o en la gestión de empresas, tiene impulso desde el marco legal tanto europeo como nacional. La reglamentación abarca estrategias generales para el desarrollo sostenible y también otras más específicas para el sector portuario. Por su parte, la legislación española también se hace eco de estas leyes y emite sus propias normativas.

Así pues, se llega a que la sostenibilidad tiene en cuenta la dimensión institucional, la económica, la social y la ambiental [3].

El aumento de la conciencia ambiental crea nuevos retos para el desarrollo de los puertos [4]. El cambio climático requiere medidas de adaptación orientadas a minimizar los impactos que afectan al transporte marítimo como, por ejemplo, el aumento del nivel del mar, el aumento de alturas de agua de inundación o la accesibilidad de los puertos y de las vías navegables. La legislación nacional e internacional, cada vez más estricta, para nuevos puertos o ampliaciones de los puertos existentes está incorporando estas cuestiones para conseguir un mínimo impacto ambiental y que las operaciones portuarias sean sostenibles a largo plazo [5]. Las regulaciones se aplican a través de un sistema de permisos en el que ciertos métodos de construcción y operación están predefinidos, incluyendo medidas de mitigación y compensación [4]. Los puertos deben involucrarse activamente en la adopción de estrategias acerca de su futuro, además de apoyar la economía regional y la calidad de vida de los trabajadores y de los ciudadanos. Las grandes y muy costosas infraestructuras que se crean para organizar las operaciones portuarias requieren acertar con la tendencia escogida. Algunas medidas que se ya han llevado a cabo para fomentar el transporte portuario sostenible a nivel internacional son [3]:

- Fomento del cabotaje. Se establecen diversas medidas incentivas, como las bonificaciones a la tasa del buque, el pasaje y la mercancía, con el fin de fomentar el tráfico marítimo de corta distancia.

- Impulso al ferrocarril. Se establecen diversas medidas para impulsar el intercambio modal entre transporte marítimo y transporte ferroviario.

- Mejora de accesos. Se incluyen los aspectos ligados a la accesibilidad terrestre del puerto en los Planes Directores.

Con el desarrollo de este artículo se pretende relacionar la sostenibilidad con la gestión a través de un estudio integrado de los indicadores de gestión de las terminales de contenedores del sistema portuario español y de los indicadores medioambientales.

\section{Los indicadores de desempeño: KPI y la sostenibilidad}

Los indicadores clave de desempeño de los procesos o KPI (Key Performance Indicator) son herramientas de gestión ampliamente utilizadas por las empresas en todo el mundo para medir y evaluar el desempeño de sus procesos y gestionarlos de la manera más eficaz y eficiente posible, con miras a la consecución de 
metas y objetivos previamente establecidos por las organizaciones. Este indicador debe ser calculado por un índice (generalmente representado por un número) que retrate el progreso del proceso en su conjunto o en parte. Para ser un indicador realista, cada KPI debe basarse en un objetivo razonable y debe cambiar con el tiempo a medida que el negocio mejora [1].

Por otro lado, el concepto de desarrollo sostenible es amplio y abstracto. Se inició como una búsqueda de compatibilidad entre el desarrollo económico, la protección y el uso adecuado de los recursos naturales. Este nuevo concepto supuso un cambio social, ambiental y económico importante al incluir cuestiones medioambientales que nunca antes habían sido debatidas. Sin embargo, esta visión se amplió a otras dimensiones, de tal forma que la definición ha evolucionado, involucrando más áreas del conocimiento. En las dos últimas décadas del S. XX, el aumento de la demanda social de una mayor transparencia en la información que suministran las organizaciones ha hecho que el desarrollo sostenible evolucione. En el caso del sector transportes, este concepto se está aplicando de forma emergente, impulsado por iniciativas que incorporan la variable ambiental y la responsabilidad social en la gestión estratégica de las empresas. El objetivo a largo plazo es el mantenimiento equilibrado de su función, buscando un desarrollo conjunto de la dimensión económica, social, ambiental e institucional [1].

Así pues, se llega a que, desde el punto de vista exclusivo de la sostenibilidad, se encuentran 4 características: Medioambiental, económica, social y gobernanza [6].

El desarrollo sostenible es un concepto amplio y abstracto que parte de la búsqueda de compatibilidad entre el desarrollo económico, la protección y uso adecuado de los recursos naturales. En el sector transportes, este concepto se está aplicando de forma emergente, impulsado por iniciativas que incorporan la variable ambiental y la responsabilidad social en la gestión estratégica de las empresas. En este contexto, merece una atención particular el transporte marítimo, donde los criterios de sostenibilidad se encuentran ya incorporados en las políticas que impulsan las Autoridades Portuarias, de forma que la sostenibilidad portuaria tiene sus raíces en las propuestas del GRI (Global Reporting Initiative) que integran las cuatro dimensiones o ejes [6] que conforman un enfoque de desarrollo sostenible, es decir, el institucional, el económico, el ambiental y el social $[7,8]$.

Los Estándares GRI crean un lenguaje común para las organizaciones y los grupos de interés, con el que los impactos económicos, ambientales y sociales de las organizaciones pueden ser comunicados y comprendidos. Los Estándares se han diseñado para fomentar la comparabilidad global y la calidad de la información sobre estos impactos y posibilitar una mayor transparencia y rendición de cuentas por parte de las organizaciones [7].

Los puertos, por el impacto que generan en las distintas ciudades donde desarrollan sus actividades y sus problemáticas específicas, deberían contar con una Guía propia, tal como ya lo hacen los aeropuertos, por ejemplo. Sin embargo, y más allá de este vacío, han existido múltiples iniciativas para reportar en el sector portuario a nivel mundial [9].

Por tanto, se debe entender la gestión sostenible como "Aquella que permite que crezca el volumen de tráfico de contenedores, graneles sólidos y líquidos, mercancía general y número de pasajeros, disminuyendo a su vez el consumo de energía y recursos naturales, el volumen de residuos generados y los impactos negativos a los sistemas sociales y ecosistemas en las áreas de influencia del puertos", Incluso se puede entender como "Un marco que permita que crezca el número del negocio portuario (contenedores, mercancía general, pasajeros), pero desvinculado de los efectos negativos a los entornos sociales y al ambiente" $[9,10]$.

De esta forma se considera que la gestión sostenible de una empresa $u$ organismo tiene como meta el mantenimiento equilibrado en el largo plazo de su función y actividad, de modo que se tengan en cuenta el efecto reciproco de su actividad con el entorno económico, social y ambiental con el que se relaciona y el efecto de su actividad con su propia estructura al tiempo que se el efecto de sus funciones y actividades en sus aspectos o dimensiones económica, social, medioambiental e institucional, buscando un desarrollo equilibrado de estas cuatro dimensiones [11].

En 1987, la Comisión Mundial sobre el Medio Ambiente y el Desarrollo fijó un ambicioso objetivo 
de desarrollo sostenible, que describió como "Desarrollo que satisface las necesidades del presente sin poner en riesgo la capacidad de las generaciones futuras para satisfacer sus propias necesidades". A través de sus actividades y relaciones, todas las organizaciones contribuyen de forma positiva y negativa al objetivo de desarrollo sostenible. Por lo tanto, las organizaciones desempeñan un papel clave en el logro de este objetivo [12]. En este estudio, teniendo presente lo dicho con anterioridad, siendo conscientes de que la sostenibilidad engloba cuatro características, se va estudiar la característica medioambiental. Desde el punto de vista ambiental se analiza: Cuáles son las presiones o impactos de la actividad sobre el medio ambiente y cuáles son las acciones para limitar el impacto.

\section{El sistema portuario español: Sostenibilidad y KPI} Señalar que, en el sistema portuario español, la gestión ambiental del puerto, está claramente condicionada por el esquema de explotación público-privada. $\mathrm{La}$ eficiencia ambiental del puerto no depende únicamente de la autoridad portuaria, sino también del rigor de la gestión ambiental realizada por concesiones, prestadores de servicios y usuarios del puerto. En general, esta competencia descansa sobre las comunidades autónomas, quienes están dotadas de un régimen sancionador que permite actuar contra posibles incumplimientos [10]. Sin embargo, las Autoridades Portuarias desempeñan un papel clave en la adecuada gestión ambiental del puerto ya que actúan como gestores de infraestructura, reguladores, coordinadores de los servicios prestados y, en especial, como líderes de la comunidad portuaria. Para poder analizar esta característica (ambiental de los puertos) es necesario evaluar una serie de factores que hagan referencia a: La gestión ambiental, la calidad del aire, la calidad del agua, la calidad acústica, los residuos, la ecoeficiencia [11].

Los indicadores de sostenibilidad ambiental constituyen una metodología para evaluar las incidencias de los procesos productivos sobre el medio ambiente. Estos indicadores permiten cuantificar el grado de responsabilidad y sostenibilidad ambiental de un individuo, organización o comunidad. $\mathrm{La}$ fuente de los datos es preferible que sea de origen público para que se garantice su objetividad, y que los datos disponibles no estén sujetos a restricciones ni sean ambiguos o poco fiables. Los indicadores que se van a seleccionar para realizar un análisis estadístico de clasificación deben reunir una serie de características [13, 14]:

- Relevantes a lo que se mide.

- Objetivos, sin ambigüedad sobre lo que es que se está midiendo.

- Auditables, para garantizar la fiabilidad y verificabilidad.

- Inequívocos, sin posibilidad para mala interpretación o re-interpretación.

- Fácilmente calculables, de tal manera que la información sea de fácil acceso.

Existen numerosos indicadores de sostenibilidad, sin embargo, se puede decir que entre lo más utilizados se encuentran: La huella ecológica, la huella de carbono, la huella hídrica y la huella social.

Inicialmente los KPI que se analizaban en los puertos estaba focalizados en el desempeño de la autoridad portuaria, sin dejar de lado estos, poco a poco se han ido introduciendo los indicadores que conciernen a la prestación de los servicios en el puerto.

La tendencia general en el análisis de la explotación de una terminal de contenedores es comparar los ratios y parámetros internacionales que aparecen en la bibliografía internacional sobre explotación y planificación portuaria, criterios con los que se explotan, y así establecer lo que una terminal se aleja o se acerca en su explotación a los valores de estos parámetros internacionales. Para la caracterización de los parámetros físicos de las terminales, se necesitan de la obtención de los datos de las propias terminales para contar con los datos reales de la propia explotación [10].

En el espacio conceptual del rendimiento portuario se emplean, habitualmente con escasa precisión, diversos términos como tráfico, capacidad, productividad, ocupación, eficiencia... A la hora de abordar una descripción precisa del estado actual de los conocimientos en la materia es conveniente plantear una ordenación terminológica de los referidos términos. Así una posible clasificación de rendimiento portuario, plantea tres tipos o categorías de medición: Mediciones de tráfico, mediciones de productividad y mediciones de utilización [1].

- Las mediciones de tráfico expresan volúmenes manipulados por unidad de tiempo, sin explicitar los recursos empleados. 
- Las mediciones de productividad expresan volúmenes manipulados (Producción) por unidad de recurso y por unidad de tiempo.

- Las mediciones de utilización son ratios (Por ejemplo, la tasa de ocupación de la línea de atraque), expresados en porcentaje, entre el uso de un determinado recurso y el máximo posible en un periodo temporal.

Así, la capacidad de una infraestructura o equipo resulta del producto de una medición de utilización por otra productividad para una unidad temporal, que será función del nivel de servicio requerido.

El último concepto incorporado en el área de rendimiento portuario, desde mediados de los noventa, es el de la literatura sobre eficiencia. La eficiencia se entiende como la capacidad de alcanzar los objetivos programados con el mínimo recurso disponible, logrando así su optimización o, alternativamente, puede expresarse como alcanzar el máximo objetivo con los recursos disponibles. El conjunto de categorías e indicadores tipo para medir el rendimiento portuario puede ser reclasificado atendiendo al uso u objetivo del correspondiente indicadores tipo [9]. Así, cabe diferenciar entre mediciones de rendimiento a corto plazo y mediciones de rendimiento a largo plazo.

Las mediciones a corto plazo (básicas o individuales) son aquellas que caracterizan la calidad de servicio prestado a un buque o vehículo de transporte terrestre concreto. Su seguimiento por parte del control de la terminal se realiza a tiempo real, de modo que paralelamente se pueden tomar decisiones para adecuar el servicio a las necesidades del cliente [7].

Las mediciones a largo plazo (Agregadas o colectivas) son aquellas que caracterizan la calidad del servicio prestado en un periodo de tiempo (mes, año). Su seguimiento y control por parte del gestor del puerto o terminal se realiza con el objetivo de la planificación de acciones a medio y a largo plazo [6].

\section{DESARROLLO METODOLÓGICO Y ANÁLISIS DE RESULTADOS}

El desarrollo metodológico de la investigación se resume en la Figura 1. Con más detalle se describe a continuación el proceso.

\section{Determinación del escenario de trabajo}

Para la realización del estudio se consideraron las terminales españolas con mayor movimiento de TEUS, empleando para ello las memorias anuales de cada Autoridad Portuaria. No obstante, dado que algunas autoridades portuarias cuentan con más de una terminal de contenedores, se consideraron las Autoridades Portuarias que mueven un mayor número de TEUS, siendo el listado, el que se muestra a continuación:

1. Autoridad Portuaria de la Bahía de Algeciras: APM, TTI.

2. Autoridad Portuaria de Valencia: Noatum Valencia, MSC, APM(TCV).

3. Autoridad Portuaria de Barcelona: APM(TCB), BEST.

4. Autoridad Portuaria de Las Palmas: OPCSA, LA LUZ.

5. Autoridad Portuaria de Bilbao: Noatum Bilbao.

6. Autoridad Portuaria de Santa Cruz de Tenerife: CAPSA, TCT.

7. Autoridad Portuaria de Vigo: TermaVi.

8. Autoridad Portuaria de Alicante: TMS.

En la Figura 2 se muestran las terminales, la obtención de los KPI de estas terminales ha resultado más

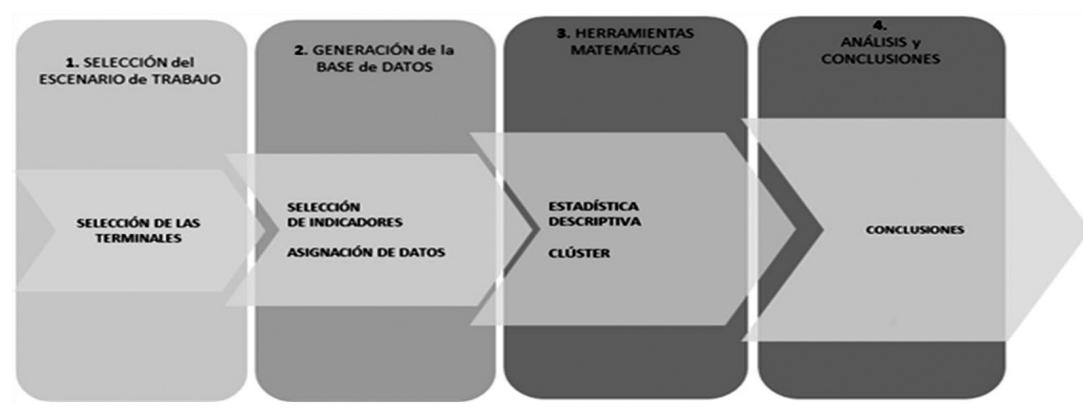

Figura 1. Esquema de la metodología empleada. 


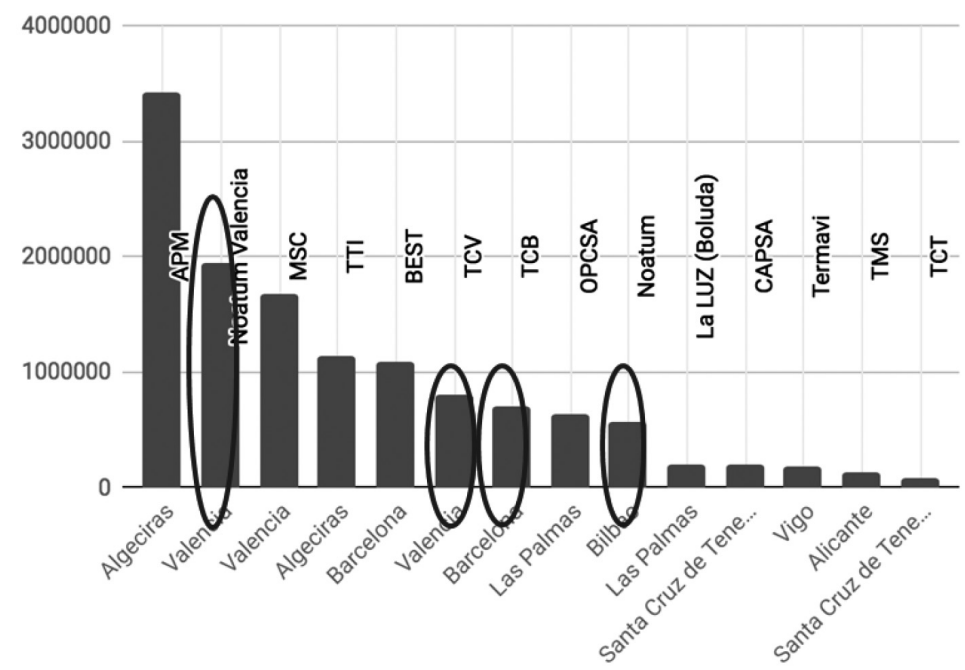

Figura 2. Terminales consideradas en el estudio. Tráficos asociados.

sencillo, pero los indicadores ambientales no han sido tan directos, puesto que muchas terminales no ofrecen los datos que se requieren para el estudio

Como ya se ha dicho con anterioridad, para poder analizar la característica ambiental de los Puertos, es necesario evaluar una serie de factores que hacen referencia a: La gestión ambiental, la calidad del aire, la calidad del agua, la calidad acústica, los residuos, la ecoeficiencia. A continuación, se va a ir desarrollando como se han obtenido los datos de los diferentes aspectos en el ámbito ambiental.

Lo primero que se lleva a cabo es clasificar las terminales según tengan un sistema de gestión ambiental EMAS o ISO 14001, según establece el Plan de Empresa 2019 de Objetivos e indicadores Medioambientales de Puertos del Estado. La norma ISO 14001 [14] es la norma internacional de sistemas de gestión ambiental (SGA), que ayuda a su organización a identificar, priorizar y gestionar los riesgos ambientales, como parte de sus prácticas de negocios habituales. La norma ISO 14001 ha sido diseñada para poder ser implementada en cualquier organización independientemente de su tamaño, sector y ubicación geográfica.

EMAS es una herramienta de uso voluntario disponible para cualquier organización que opere en algún sector económico de la Unión Europea o fuera de la Unión Europea y que quiera asumir una responsabilidad ambiental y económica, mejorar su comportamiento ambiental y comunicar sus resultados ambientales a la sociedad y a las partes interesadas en general. Se llega a que, de las 14 terminales del estudio, únicamente 4 terminales tienen el sistema de gestión EMAS [15].

\section{Generación de la base de datos}

Cada base de datos se compone de una o más tablas que guarda un conjunto de datos. Cada tabla tiene una o más columnas y filas. Las columnas guardan una parte de la información sobre cada elemento que se quiere guardar en la tabla, cada fila de la tabla conforma un registro. Con los datos de los indicadores que se han descrito en el punto anterior se ha desarrollado la base de datos de manera que permite guardar grandes cantidades de información de forma organizada para que luego se pueda encontrar y utilizar fácilmente. Por lo tanto, se ha generado una serie de datos organizados y relacionados entre sí, los cuales son recolectados y explotados por los sistemas de información.

Los indicadores ambientales empleados son la calidad acústica, la calidad del aire, la gestión de los residuos y la eficiencia energética. Así, considerando las 14 terminales, los indicadores se obtienen:

La calidad acústica: Para la adquisición de este indicador se realiza una estimación global de los resultados de los mapas acústicos de cada ciudad. Por lo tanto, como únicamente se tienen los mapas por ciudades este valor en vez de hacerlo por terminales 
se va a referenciar a las autoridades portuarias. En los mapas acústicos la información viene detallada de la siguiente manera:

- Lday (Ld) es el nivel sonoro medio a largo plazo ponderado A definido en la norma ISO 1996-2: 1987 [16], determinado a lo largo de todos los períodos día de un año.

- Levening (Le) es el nivel sonoro medio a largo plazo ponderado A definido en la norma ISO 1996-2: 1987 [16], determinado a lo largo de todos los períodos tarde de un año.

- Lnight (Ln) es el nivel sonoro medio a largo plazo ponderado A definido en la norma ISO 1996-2: 1987 [16], determinado a lo largo de todos los períodos noche de un año.

- Lden (Indicador de ruido día-tarde-noche) es el indicador de ruido asociado a la molestia global, expresado en decibelios, el cual se determina aplicando la ec (1):

$$
\begin{gathered}
\text { Lden }=10 \log (1 / 24)(12 \times 10 \text { Lday/10 + 4x } 10 \\
(\text { Levening + 5)/10 + } 8 \times 10(\text { Lnight }+10) / 10)
\end{gathered}
$$

Donde: Al día le corresponden 12 horas, a la tarde 4 horas y a la noche 8 horas. Los valores horarios de comienzo y fin de los distintos períodos son 7,0019,00 para Ld, 19,00-23,00 para Le y 23,00-7,00 para $\mathrm{Ln}$, hora local.

De esta forma se obtiene los decibelios medidos en la zona cercana al puerto, y con ello a la terminal, pues si los decibelios medidos en la autoridad son
60, también lo serán en cada una de las terminales de esa autoridad, por lo tanto, se extrapola para cada terminal (Tabla 1).

La calidad del aire: En este caso, se obtiene un valor que oscila entre 0 y 300 según el ICA (Índice de Calidad del Aire). El índice de calidad del aire, es un indicador que sirve para informar de la calidad del aire de una manera clara y sencilla. Para obtener el ICA se dispone de una red de control y vigilancia que mide en tiempo real una serie de parámetros, tales como los contaminantes SO2, NOx, CO, PM10 y O3. El conjunto de valores que el ICA puede tomar se agrupa en intervalos a los que se les asocia una trama o color característico de la calidad del aire de una zona determinada. Está dividido en cinco categorías, que definen los estados de calidad de aire en: Muy Buena, Buena, Mejorable, Mala y Muy Mala (Figura 3).

Los resultados obtenidos para cada Autoridad Portuaria son los que se recogen en la Tabla 2.

Como el ICA se obtiene en un momento determinado, para la obtención de los valores definitivos en la presente investigación, se hace una media con diferentes números del ICA lo largo de varios días y finalmente se obtienen los siguientes datos:

Residuos: Para la obtención de un valor de este parámetro que permitiera comparar unas autoridades

Tabla 1. Decibelios por terminal.

\begin{tabular}{|l|l|c|}
\hline Autoridad Portuaria & Nombre de terminal & Calidad acústica \\
\hline Algeciras & APM & 52,5 \\
\hline Valencia & Noatum Valencia & 60 \\
\hline Valencia & MSC & 60 \\
\hline Algeciras & TTI & 52,5 \\
\hline Barcelona & BEST & 62,5 \\
\hline Valencia & TCV & 60 \\
\hline Barcelona & TCB & 62,5 \\
\hline Las Palmas & OPCSA & 65 \\
\hline Bilbao & Noatum & 52,5 \\
\hline Las Palmas & La LUZ (Boluda) & 65 \\
\hline Sta. Cruz de Tenerife & CAPSA & 62,5 \\
\hline Vigo & Termavi & 65 \\
\hline Alicante & TMS & 62,5 \\
\hline Sta. Cruz de Tenerife & TCT & 62,5 \\
\hline
\end{tabular}




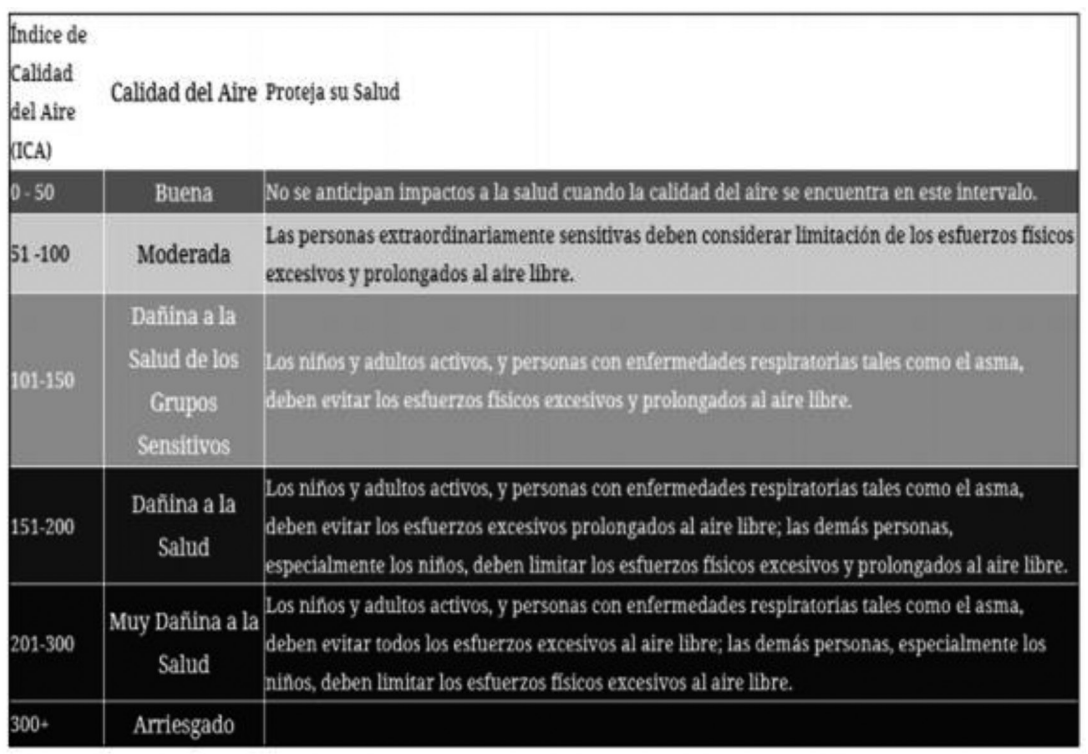

Figura 3. Niveles de calidad del aire.

Tabla 2. Calidad del aire por autoridad portuaria.

\begin{tabular}{|l|c|c|c|c|}
\hline Autoridad Portuaria & $\begin{array}{c}\text { Calidad del aire } \\
\text { (según el ICA, valores de 0-300) }\end{array}$ & $\begin{array}{c}\text { Total } \\
\text { Ecoeficiencia }\end{array}$ & $\begin{array}{c}\text { Toneladas } \\
\text { movidas }\end{array}$ & Kwh/tn \\
\hline Valencia & 60 & 12176400,99 & 96861660 & 0,13 \\
\hline Las Palmas & 51 & 6506850,969 & 70995541 & 0,09 \\
\hline Algeciras & 40 & 8844987,538 & 47512963 & 0,19 \\
\hline Alicante & 36 & 5687019,973 & 20192621 & 0,28 \\
\hline Sta. Cruz de Tenerife & 36 & 6891907,961 & 31948743 & 0,22 \\
\hline Barcelona & 35 & 4328639,571 & 12957892 & 0,33 \\
\hline Vigo & 30 & 4095002,117 & 3861603 & 1,06 \\
\hline Bilbao & 29 & 1821944 & 3442581 & 0,53 \\
\hline
\end{tabular}

portuarias con otras y saber cuál está siendo la dinámica general de las autoridades españolas en materia de la gestión de sus residuos, se ha cogido la lista de las 10 medidas que propone la memoria anual de puertos del Estado en su dimensión ambiental y se ordenan las autoridades en función del número de medidas que tienen implementadas. Para conocer el número de medidas que cada autoridad pone en práctica, se mira en la memoria de sostenibilidad de cada una de la autoridad portuaria.

En el presente trabajo se va a realizar una primera estimación estableciendo un peso de 1 a cada una de las medidas, sin embargo, cabe decir que un valor ponderado para el cumplimiento, podría ser objeto de otra investigación.
Ecoeficiencia: Para el cálculo de la ecoeficiencia, se ha calculado el consumo eléctrico $(\mathrm{kWh})$ y el consumo de combustible (L) y se obtiene un total, gracias a la equivalencia de $1 \mathrm{kWh}=0,0991 \mathrm{~L}$ de gasoil Una vez que se tiene todo los litros de combustibles pasados a kWh se suman los $\mathrm{kWh}$ debidos al consumo eléctrico y las kWh debidos al consumo de combustible, con estos $\mathrm{kWh}$ totales se obtiene una relación entre los kWh de la autoridad y las toneladas totales movidas por la autoridad, y se obtienen los valores incluidos en la Tabla 2.

El siguiente paso consiste en definir los KPI a estudiar. La tendencia general en el análisis de la explotación de una terminal es comparar los ratios y parámetros internacionales, y establecer lo que 
una terminal se aleja o acerca en su explotación a los valores de estos parámetros internacionales. En esta investigación se han cogido los KPI que estableció la IAPH (International Association of Ports and Harbors) como los indicadores más adecuados para poder comparar unas terminales con otras. Del estudio realizado por la IAPH se obtienen los KPI que se van a utilizar: TEU por metro de muelle, TEU por hectárea, TEU por muelle grúa, TEU por RTG, TEU por Straddle Carrier.

Aunque en el estudio realizado por la IAPH también incluía dentro de estos indicadores el TEU por estibados y TEU por escala, en el presente trabajo no se van a tener en cuenta por la dificultad que se ha tenido a la hora de encontrar los datos no llegando a ningún valor con el que poder trabajar.

- TEU por metro de muelle: Indica la productividad del muelle. Cuanto mayor sea, mayor es la productividad de la terminal

- TEU por hectárea: Expresa la productividad de la explanada.

- TEU por grúa de muelle: Indica la productividad operativa de las grúas que tiene la terminal en el muelle.

- TEU por RTG: Expresa la productividad operativa de los RTG.

- TEU por Straddle Carrier: Indica la productividad operativa de los Straddle Carrier. De las 14 terminales del estudio únicamente cuatro terminales tienen Straddle Carrier en sus equipos. El resto optan por RTG

\section{Herramientas metodológicas}

Para llevar a cabo el presente estudio se han utilizado dos herramientas: Por un lado, la estadística descriptiva y por otro lado el análisis Clúster o de conglomerados. A continuación, se definen algunos conceptos de teoría de estadística descriptiva y de análisis de conglomerados, y se explica cómo se ha llevado a cabo la normalización de los indicadores para su posterior comparación.

\section{Estadística descriptiva}

La investigación cuya finalidad es: El análisis o experimentación de situaciones para el descubrimiento de nuevos hechos, la revisión o establecimiento de teorías y las aplicaciones prácticas de las mismas, se basa en los principios de observación y razonamiento y necesita en su carácter científico el análisis técnico de Datos para obtener de ellos información confiable y oportuna. Este análisis de datos requiere de la estadística como una de sus principales herramientas, por lo que los investigadores de profesión y las personas que de una y otra forma la realizan requieren además de los conocimientos especializados en su campo de actividades, del manejo eficiente de los conceptos, técnicas y procedimientos estadísticos.

\section{Análisis Clúster}

En cuanto al análisis de conglomerados, se puede resumir diciendo que trata de obtener clasificaciones de grupos homogéneos. Los trabajos de Sokal y Sneath marcaron el inicio de los agrupamientos, que se han extendido por todos los ámbitos científicos [17].

En el caso de los análisis de posicionamiento y jerarquización el análisis por agrupamiento tipo clúster, resulta muy útil por su versatilidad y sencillez. Este tipo de análisis permite aproximaciones a tipologías del universo del estudio desde varios puntos de vista según su relación simultánea en las dimensiones que se han definido.

A continuación, se presenta los resultados de las gráficas que se han realizado mediante estadística descriptiva, así como los resultados obtenidos mediante el análisis clúster. Se han realizado dos tipos de gráficas: En las primeras, en eje de abscisas se coloca las terminales ordenadas de mayor a menor en función del indicador de productividad (TEU/m, TEU/ha, TEU/grúa, TEU/RTG, TEU/ $\mathrm{SC})$ y se tiene un doble eje de ordenadas, en lado derecho se colocan los KPI y en el izquierdo los indicadores ambientales. De esta forma se tienen 4 gráficas (Residuos, calidad del aire, calidad acústica y eficiencia energética) para cada uno de los KPI, este tipo de gráficas permite encontrar la relación entre unos indicadores y los otros. En las segundas, son gráficas de pares de puntos; donde se han ordenado ambos indicadores (Ambientales y de productividad) en función de los TEU movidos, a cada valor del indicador de productividad le corresponde un valor del indicador ambiental. En el eje de las abscisas se colocan los indicadores de productividad y en el de ordenadas el indicador ambiental correspondiente.

\section{Estudio mediante estadística descriptiva}

Se ha realizado un estudio de estadística descriptiva mostrándose en las Figura 4, Figura 5 y Figura 6 sólo se muestran algunos ejemplos representativos. 


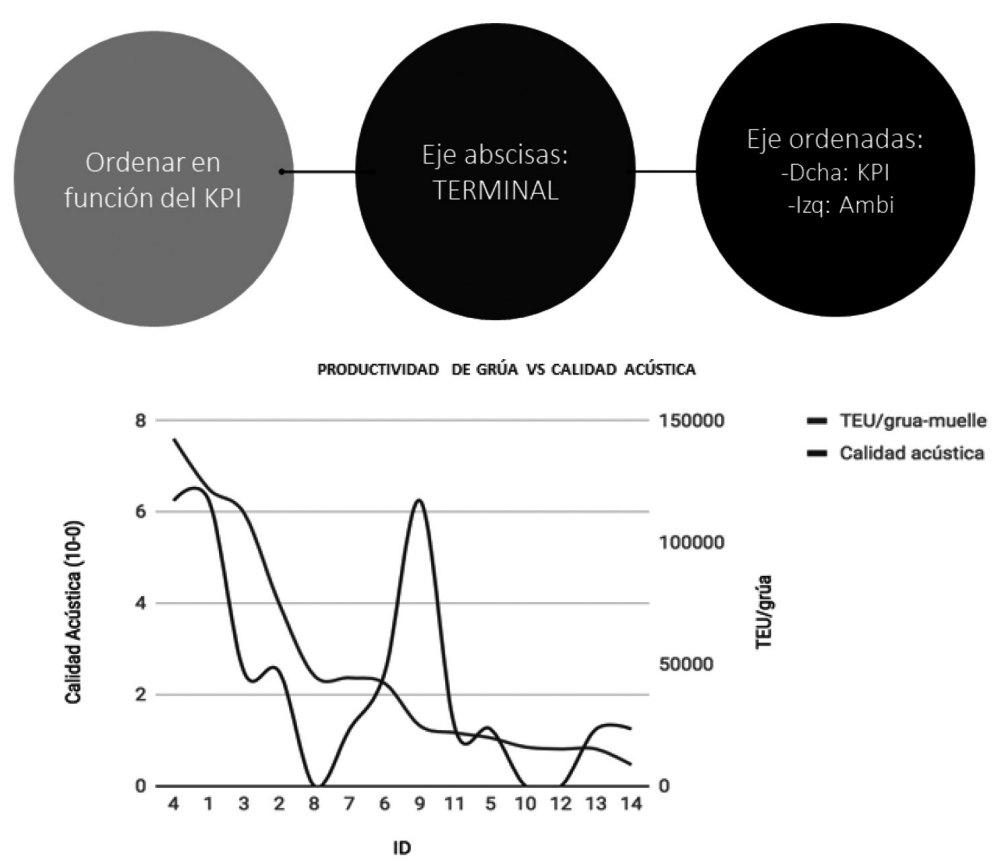

Figura 4. Resultado 1 de calidad acústica obtenido con estadística descriptiva.

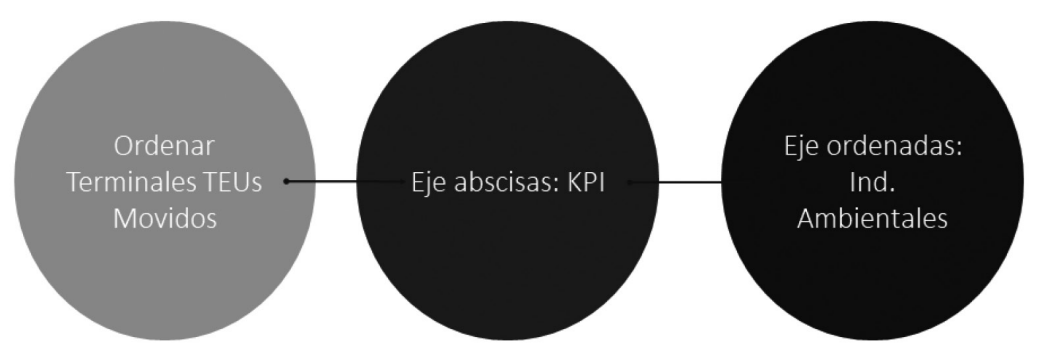

Figura 5. Resultado 2 de calidad acústica obtenido con estadística descriptiva.

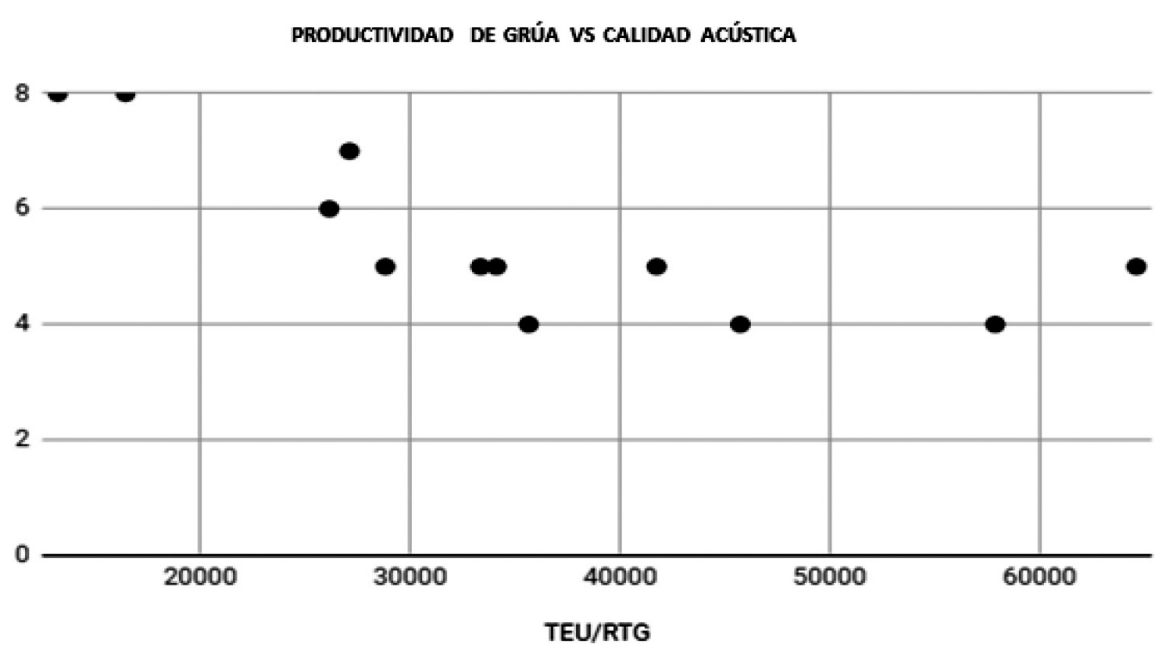

Figura 6. Resultado de residuos obtenido con estadística descriptiva. 
Se puede decir que existe una relación directa entre la productividad operativa medida en TEU/grúa y parámetro ambiental de la calidad acústica. A medida que disminuye la productividad operativa, disminuye también la calidad acústica.

\section{Análisis clúster}

Tras la realización del análisis clúster los resultados obtenidos son los que se muestran en la Figura 7.

\section{Análisis de resultados}

Dado que el objetivo del presente estudio es enfrentar los indicadores ambientales y los KPI y ver si se puede sacar alguna relación, es necesario que para ello estén todos expresados en términos unitarios. Para relacionar los KPI con los indicadores ambientales, se ha cogido cada indicador KPI y se ha enfrentado con cada uno de los indicadores ambientales. De esta forma se obtienen 4 gráficas por cada KPI, un total de 20 gráficas por cada uno de los estudios.

Se optó por realizar gráficas de doble eje, donde las abscisas fueran las terminales y en el eje derecho de ordenadas el KPI y en el eje izquierdo de ordenadas el indicador ambiental. En este caso las terminales no se ordenan por TEUs movidos, sino que se ordenan de mayor a menor en función del parámetro KPI.Y el indicador ambiental se ordena también en función
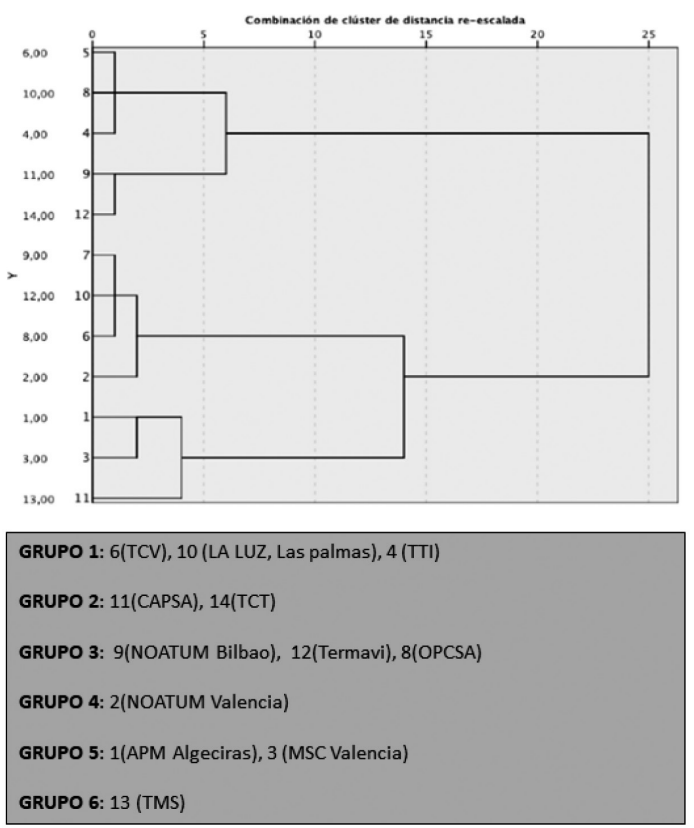

Figura 7. Resultados del análisis clúster. del KPI correspondiente. Como los KPI vienen en expresados en términos unitarios y un valor alto del KPI tiene una connotación positiva, pues nos indica que la productividad es alta, es preciso transformar los indicadores ambientales en unitarios y demás expresar en valores altos lo positivo y en valores bajos lo que es negativo. A continuación, se muestra como se ha expresado cada indicador ambiental para llegar a lo que se buscaba.

En el caso de las 14 terminales, la calidad acústica se ha obtenido como los decibelios medidos en esa zona. Cuanto mayor es el valor, menor es la calidad acústica y como se quiere expresar de forma inversa. Un valor alto para mayores calidades acústicas, se ha escalado dando a los 65 decibelios el valor 0 (lo peor) y a los 45 decibelios el valor 10 (Lo mejor).

Por otro lado, la calidad del aire viene expresada como un valor del 0 al 300, donde 300 es la peor calidad del aire y el 0 lo mejor. Como en el caso anterior, en el presente estudio se busca lo contrario, valores altos para calidades buenas y valores bajas para calidades malas. En este caso, simplemente se ha invertido los valores el 0 pasa a ser el 300 y el 300 el 0 y los demás números se obtienen de manera proporcional.

Para la gestión de los residuos se recoge el número de medidas propuestas por puertos del Estado para una correcta gestión de los residuos, en este caso un mayor número de medidas implantadas sí que representa una mayor calidad en la gestión de los residuos, por lo tanto, este indicador, se queda como inicialmente estaba expresado.

Finalmente, la eficiencia energética viene dada en $\mathrm{kWh}$ consumidos, como se quiere dar los valores altos significado positivo y los más bajos negativo, se expresa la eficiencia energética en TEU/kWh y de esta forma a más $\mathrm{kWh}$ menor es el parámetro que es lo que se buscaba, pues a más kWh menor es la eficiencia.

Para el análisis de la estadística descriptiva, se han seleccionado de las 16 combinaciones de parámetros de productividad con parámetros medioambientales 3 de las que pueden ser más ilustrativas. En la selección se ha tenido en cuenta la relación lógica que a priori pueden tener los parámetros y los propios resultados de la comparativa según la 
gráfica de pares de puntos, poniendo en abscisas los indicadores de productividad y en ordenadas los indicadores ambientales.

\section{- Productividad del muelle VS Residuos}

Por la forma en que se han establecido los parámetros medioambientales con respecto a los residuos, la nube de puntos que se visualiza en esta gráfica supone una comparativa entre la productividad de muelle de una terminal con el grado de compromiso medioambiental en relación a la gestión de los residuos que tiene la autoridad portuaria. Se observa que para menos productividad de muelle hay una mayor dispersión, se pueden encontrar para unos valores similares de productividad de muelles unos grados de compromisos medioambientales en la gestión de residuos muy diferentes; mientras que para productividad de muelle medias o altas los valores del grado de compromiso se sitúan en unos valores intermedios más similares.

- Productividad de la explanada VS Calidad acústica.

No se aprecia en la nube de puntos generada una relación entre ambos parámetros. Hay que tener en cuenta que los valores de la calidad acústica, son valores que indican la calidad acústica general de la zona donde está la terminal, no responden a mediciones realizadas en la propia terminal.

- Productividad de RTG VS Calidad del aire. La nube de puntos que se muestra en la gráfica, indica la independencia entre la calidad el aire y la productividad de RTG. La relación entre los parámetros es dispersa, esto se puede deber en parte, a que lo que se recoge son los valores generales de la calidad del aire de la zona y no de mediciones específicas de la propia terminal.

En el clúster hecho únicamente con los indicadores de productividad se forman seis grupos, que a su vez se pueden agrupar en tres (El 1 y el 2, el 3 y el 4 y finalmente el 5 y el 6 ).

- Grupo 1: 6 (TCV Valencia), 10 (LA LUZ, Las Palmas), 4 (TTI Algeciras).

- Grupo 2: 11 (APM Algeciras), 14 (TCT Santa Cruz de Tenerife).

- Grupo 3:9 (NOATUM Bilbao), 12 (TERMAVI Vigo), 8 (OPCSA Las palmas).

- Grupo 4: 2 (NOATUM Valencia).

- Grupo 5: 1 (APM Algeciras), 3 (MSC Valencia).

- Grupo 6:13 (TMS Alicante).
El resultado indica que la formación de grupos en relación con la productividad no depende del resultado operativo del tráfico total movido por la terminal, que a su vez tiene relación con el tamaño de la terminal. Así, por ejemplo, en el grupo 1, tenemos una terminal que mueve unos 800000 , otra que mueve alrededor de 200000 y la tercera que mueve un millón de TEUs.

A partir de los resultados obtenidos del estudio se puede concluir:

- No se han encontrado antecedentes en el sector que relacionan los parámetros de productividad con los ambientales. Mientras en la parte de productividad, como es un tema que influye de manera más directa e inmediata en los resultados económicos, las propias terminales y el sector en general, desde hace ya mucho tiempo, han realizado mediciones de los KPI, por el contrario, en relación con los ambientales, con excepción de los casos de terminales que han implantado sistema de gestión medioambiental tipo EMAS, no se realizan mediciones de parámetros medioambientales básicos; mucho menos si relacionan estos con otros parámetros como los de productividad.

- La generación de la base de datos ha resultado muy difícil. Los indicadores de productividad (KPI) se basan en parámetros de la propia terminal por lo tanto generar la base de datos ha sido sencillo. Sin embargo, los indicadores ambientales no se encuentran a nivel de terminal (Excepto en las terminales que disponen de sistema de gestión ambiental EMAS) y se ha tenido que estimar haciendo una primera aproximación, asignando a la terminal los parámetros de la autoridad portuaria donde se encuentra la terminal o de la zona. Además, la dificultad se acentúa al tratar con memorias no homogeneizadas y donde la información relevante para la obtención de estos parámetros generalmente no está disponible.

\section{CONCLUSIONES}

La principal limitación del estudio ha sido la diferencia significativa entre la disponibilidad de unos y otros parámetros. Mientras que cuentan con parámetros de productividad específicos de cada una de las terminales, no existen en general valores de 
parámetros medioambientales que permitan obtener unos indicadores medioambientales específicos de cada terminal.

Los KPIs se obtienen a partir de datos físicos de la terminal (longitud de muelle, superficie de explanada, número de equipos) y resultados operativos (número de contenedores movidos), valores disponibles en todas las terminales. Los indicadores medioambientales requieren disponer no solo de los resultados operativos sino también de mediciones de parámetros ambientales, como puede ser, el nivel acústico, la calidad del aire, la cantidad de residuos tratadas... Salvo en los casos de terminales que han desarrollado algún sistema de gestión ambiental, no se tienen disponibilidad de estos parámetros. Por lo que la relación entre los KPIs y los indicadores ambientales se ha tenido que hacer asignando a los últimos los mejores valores disponibles en este momento, ya sean valores correspondientes a la autoridad portuaria donde está la terminal o la zona en la que está ubicada la terminal. Algunas terminales que cuentan con sistema de gestión ambiental EMAS sí disponen de mediciones y datos ambientales más detallados, pero son solo cuatro del total de las catorce seleccionadas, lo que no permite un análisis estadístico y de clúster, sería necesaria una muestra más significativa del conjunto.

Por tanto, la limitación de este estudio es que se trata de una primera estimación que permite reflexionar sobre la relación que pueda existir entre los KPI y los indicadores ambientales, pero que necesitará un análisis más detallado, en el cual, los indicadores medioambientales se obtengan a partir de parámetros medidos en la terminal.

La aportación de este estudio, no son los resultados obtenidos en sí mismos en la comparativa realizada entre parámetros de productividad y los indicadores medioambientales de las terminales, pues estos resultados adolecen de las limitaciones referidas a los indicadores ambientales. No obstante, propone una metodología de comparación entre ambos indicadores y define unos indicadores relevantes a considerar tanto en lo relativo a la productividad como al comportamiento medioambiental.

En cuanto a la prospectiva de los indicadores a considerar en terminal de contenedores, se debería buscar un enfoque integral que incluya una visión relacionada entre los diversos parámetros, tanto de productividad como de comportamiento ambiental u otros. Teniendo en cuenta la falta de disponibilidad de parámetros medioambientales que ha aflorado en este estudio, se sugiere que se incentive a las terminales para que dispongan de esos parámetros en el futuro, y parece que la forma práctica más inmediata es incentivar a las terminales para que dispongan de un sistema de gestión medioambiental EMAS o similar.

\section{REFERENCIAS}

[1] B. Molina Serrano, N. González-Cancelas y F. Soler-Flores. "Gestión de la sostenibilidad portuaria basada en un modelo de redes bayesianas. Aplicación al sistema portuario español". Ingeniare. Revista chilena de ingeniería. Vol. $26 \mathrm{~N}^{\circ}$ 4, pp. 631-644. 2018. ISSN 0718-3305. DOI: $10.4067 /$ S0718-33052018000400631.

[2] O. Pinheiro de Lima, S. Breval Santiago, C.M. Rodríguez Taboada y N. Follmann. "Una nueva definición de la logística interna y forma de evaluar la misma". Ingeniare. Revista chilena de ingeniería. Vol. $25 \mathrm{~N}^{\circ} 2$, pp. 264-276.2017. ISSN 0718-3305. DOI: 10.4067/S0718-33052 017- 000200264.

[3] B. Molina Serrano, M.N. González Cancelas, F. Soler Flores, Francisco and A. Camarero Orive. "Classification and prediction of port variables using bayesian networks". Transport Policy. Vol. 67, pp. 57-66. 2017. DOI: 10.1016/j.tranpol.2017.07.013.

[4] M. Feitó Cespón, R. Cespón Castro y M.A. Rubio Rodríguez. "Modelos de optimización para el diseño sostenible de cadenas de suministros de reciclaje de múltiples productos". Ingeniare. Revista chilena de ingeniería. Vol. $24 \mathrm{~N}^{\circ}$ 1, pp. 135-148. 2016. ISSN 0718-3305. DOI: $10.4067 /$ S0718-33052016000100013.

[5] O. Salgado Oportus y P. Cea Echeverría. "Análisis de la conectividad externa de los puertos de Chile como un factor de competitividad". Ingeniare. Revista chilena de ingeniería. Vol. $20 \mathrm{~N}^{\circ} 1$, pp. 25-39. 2012. ISSN 0718-3305. DOI: $10.4067 /$ S0718-33052012000100004.

[6] B. Molina Serrano, N. González-Cancelas y F. Soler-Flores. "Hacia la sostenibilidad portuaria 
mediante modelos probabilísticos: Redes bayesianas". Informes de la Construcción. Vol. $70 \mathrm{~N}^{\circ}$ 549, pp. 244-255.2018. ISSN-L: 0020-0883. DOI: 10.3989/id.54678.

[7] A.E. Santos Martín, N. González-Cancelas, B. Molina Serrano and F. Soler-Flores. "Towards the sustainability of the spanish port system through the business observation tool". In Proceedings of the Institution of Civil Engineers-Maritime Engineering, pp. 1-21. 2020. DOI: 2110.1680/ jmaen. 2020.25.

[8] J. Bautista Mendo, A. Camarero-Orive, N. González-Cancelas and B. Molina Serrano. "Update of the strategic margo for the Spanish port system using a SWOT analysis". Cuadernos de administración. Vol. 36 Issue 68, pp. 96-111. 2020. DOI: 10.25100/cdea. v36i68. 9459.

[9] T. Samanés, N. González-Cancelas y B. Molina Serrano. "Integración de indicadores medioambientales e indicadores de desempeño operacional en terminales de graneles sólidos sucios del sistema portuario español”. Rect@ . Revista Electrónica de Comunicaciones y Trabajos de ASEPUMA. Vol. 20 pp. 77 a 93. 2019. DOI: 10.24309/recta.2019.20.1.05

[10] N. González-Cancelas, B. Molina Serrano and F. Soler-Flores. "Study to improve the digitalization of the Spanish port system through an affinity diagram". Journal of Maritime Transport and Logistics. Vol. 1 $\mathrm{N}^{\mathrm{o}}$ 2, pp. 51-68. 2020.
[11] B. Molina Serrano, N. González-Cancelas y F. Soler-Flores. "Gestión de la sostenibilidad portuaria basada en un modelo de redes bayesianas". Ingeniare. Revista Chilena de Ingeniería. Vol. $26 \mathrm{~N}^{\circ}$ 4, pp.631-644. 2018. DOI: $10.4067 /$ S0718-33052018000400631.

[12] Comisión Mundial sobre el Medio Ambiente y el Desarrollo. "Nuestro Futuro Común". Oxford, Oxford University Press, pp. 43. 1987.

[13] O.A. Kent, R.R. Chivukula, M. Mullendore, E.A. Wentzel, G. Feldmann, K.H. Lee, S. Liu, S.D. Leach, A. Maitra and J.T. Mendell. "Repression of the miR-143/145 cluster by oncogenic Ras initiates a tumorpromoting feed-forward pathway". Genes \& development. Vol. $24 \mathrm{~N}^{\circ} 24$, pp. 2754-2759. 2010. DOI: 10.1101/gad.1950610.

[14] N. González-Cancelas, B. Molina Serrano, F. Soler-Flores and N. C-Majolero. "Diagnosis of the digitalization of the Spanish ports: End to End tool". World Scientific News. Vol. 155, pp. 47-65. 2021.

[15] UNE-EN ISO 14001:2015. "Sistemas de gestión Ambiental". AENOR. Asociación Española de Normalización y Certificación. 2015.

[16] UNE-EN ISO 1996-2: 1987."Descripción y medida del ruido ambiental". AENOR. Asociación Española de Normalización y Certificación. 1996.

[17] R.R. Sokal and P.H.A. Sneath. "Principles of numerical taxonomy". W.H. Freeman \& Co. New York, USA. 1963. 